\title{
Significance of heparanase in metastatic lymph nodes of cervical squamous cell cancer
}

\author{
BIN HU $^{1 *}$, QING WANG ${ }^{2}$, YINGYING SHI $^{1}$, SHUFANG LU $^{1}$, HONGJIE QU $^{1}$, LU WANG $^{1}$ and JINQUAN CUI $^{1}$ \\ ${ }^{1}$ Department of Gynaecology and Obstetrics, The Second Affiliated Hospital of Zhengzhou University, \\ Zhengzhou, Henan 450014; ${ }^{2}$ Department of Gynecology, The First People's Hospital of Yunnan Province, \\ Kunming, Yunnan 650032, P.R. China
}

Received May 12, 2015; Accepted June 17, 2016

DOI: $10.3892 / \mathrm{ol} .2017 .5804$

\begin{abstract}
The aim of this study was to explore the expression of heparanase (HPA) in metastatic lymph nodes (LNs) of cervical cancer and to evaluate HPA as a marker of micro-metastasis of LNs. Immunohistochemistry was performed to detect the expression of HPA in 53 cases with metastasis of LNs (group A) and 49 cases without (group B). Scoring was determined based on the intensity of immunostaining and the size of the staining area. Three points or higher score was considered as positive. Among all cases, the positive rate of HPA was $76.5 \%$ in primary lesions and $84.9 \%$ in both primary lesions and metastatic LNs in group A. In group B, the rates were $67.3 \%$ in primary lesions and $8.2 \%$ in metastatic LNs. The expression of HPA in group A was significantly higher than that in group $\mathrm{B}(\mathrm{P}<0.05)$. Compared with stage IA-IB and well-differentiated and non-metastatic LNs, the LNs of stage IIA and moderately/poorly differentiated and metastatic LNs expressed higher HPA $(\mathrm{P}<0.05)$. The overall 5-year survival rate was $73.3 \%$ and the median overall survival time (MOS) was 49.0 months. The MOS of the two groups was 36.0 and 58.5 months, respectively $(\mathrm{P}=0.023)$; the MOS of patients with positive HPA expression was distinctly lower than that of negative patients $(\mathrm{P}=0.040)$. Clinical staging, degree of differentiation, lymph node metastasis and expression of HPA notably affected patient prognosis; lymph node metastasis and expression of HPA were independent risk factors affecting patient prognosis $(\mathrm{P}<0.05)$. Our study demonstrated that high-level expression of HPA in cervical cancer was involved in LN metastasis, further impacting on patients' long-term survival. The clinical value of HPA requires further in-depth study.
\end{abstract}

Correspondence to: Professor Jinquan Cui, Department of Gynaecology and Obstetrics, The Second Affiliated Hospital of Zhengzhou University, 8 Jingba Road, Jinshui, Zhengzhou, Henan 450014, P.R. China

E-mail: jinquan_cui@163.com

*Contributed equally

Key words: heparanase, metastatic lymph nodes, cervical cancer

\section{Introduction}

Cervical cancer is the most common female genital tract tumor in developing countries, with patients eventually succumbing to tumor invasion and metastasis. Lymph node (LN) metastasis is the most frequent route of metastasis in cervical cancer, and this is also a vital indicator in predicting the prognosis of patients and identifying patients requiring postoperative adjuvant therapy with radiation therapy (RT) and/or chemotherapy. Studies have revealed that the pelvic LN metastasis rate of early stage cervical cancer [International Federation of Gynecology and Obstetrics (FIGO) stage IA1-IB1] is 0\%-29.3\%, whereas the rate of locally advanced patients (FIGO stage IB2-IIB) is as high as $12 \%-61.8 \%(1,2)$. The 5-year survival rate of patients with LN involvement decreases from $85 \%$ to $50 \%$ (3). The number of metastatic pelvic and abdominal LNs is associated with patients' long-term survival (4). Radical hysterectomy and LN dissection is the main treatment of early cervical cancer. However, LNs dissected in this process are mostly non-metastatic, and thus may result in unnecessary intraoperative and postoperative complications. Conversely, certain micro-metastatic LNs are not correctly diagnosed or do not receive proper postoperative adjuvant treatment, and in consequence may be the main cause of rapid relapse. Even when LNs are negative, the recurrence rate of surgically treated patients reaches $10-15 \%$, among which the majority suffer from pelvic relapse $(1,5)$. The malignant transformation and lymphatic drainage of human papillomavirus (HPV)-infected atypical cells most probably account for the relapse of patients with negative LNs $(2,6,7)$.

HPV infection is the most commonly observed sexually transmitted disease $(8,9)$, and is responsible for $99 \%$ cases of cervical cancer. More than 200 types of HPV have been identified currently, of which over 40 types have been reported to be associated with reproductive tract infection, including 18 types which are directly connected with the occurrence of cervical cancer, 15 types that have been confirmed to be high-risk types that cause the disease, another 3 clinically suspected high-risk types and 13 identified low-risk types (10-12). Other than cervical cancer, HPV also leads to cancer of the anus, oral cavity and esophagus. Although the mechanism by which HPV causes cancer is not fully understand, evidence has indicated that heparanase (HPA) is a significant molecule in this 
mechanism, and that the high-risk HPV E6 oncogene is capable of inducing overexpression of HPA through a p53-dependent mechanism $(13,14)$.

HPA is a single mammalian endoglycosidase, whose activity is observed in white blood cells, mast cells, placenta tissues, neutrophilic granulocytes and macrophagocytes. It is associated with cancer progression and aggressive behavior (15-17). A previous study demonstrated that the expression of HPA is associated with cancer formation and progression in acute leukemia, bladder cancer, brain tumor, breast cancer, colon cancer, gastric cancer, esophagus cancer among others. For this reason, HPA has become a promising molecule of tumor-targeted therapy, and a variety of anti-heparanase inhibitors have been developed for clinical trials, among which P188 is in phase III clinical trials (18). Research has identified that high expression of HPA is involved in lymphatic transfer, distant metastasis and poor clinical prognosis of diverse malignant carcinomas including cervical cancer. In 2003, Shinyo et al (19) verified for the first time that the expression of HPA mRNA was promoted in advanced cervical cancer, and patients with vascular and LN involvement demonstrated an extremely high level of HPA, which was due to the close correlation between HPA expression and tumor microvascular density. These authors also confirmed that disease-free survival (DFS) and overall survival in HPA-positive patients was notably lower than in HPA-negative patients, and multiple analysis indicated that HPA expression was an independent prognostic factor. It was affirmed though immunohistochemistry that the rate of positive HPA protein expression in cervical cancer patients was $63.3 \%$, and that the expression level is correlated with tumor size and clinical stage. Overexpression of HPA inhibited the apoptosis of cervical cancer cell lines in vitro and promoted their proliferation and growth (20).

On the basis of the above findings, we may conclude that HPA has a close connection with the occurrence, progression and LN metastasis of cervical cancer. However, to date, no research on HPA expression in cervical cancer metastatic LNs has been identified, and the effect of LN metastasis of cervical cancer patients caused by abnormal HPA expression still lacks evidentiary support. To explore the role of HPA in lymphatic metastasis and patients' clinical prognosis, we study the expression of HPA in sentinel LNs of cervical cancer and investigate clinicopathological features of the tumor and patient prognosis. We reveal that the rate of HPA-positive expression in pathologically confirmed metastatic LNs is equivalent to that in the primary lesion, and a significant reduction in the recurrence rate and long-term survival rate is identified in patients with positive HPA expression in LNs. Our study proposes HPA as a significant marker for the diagnosis of micro-metastasis of LN in cervical cancer and a theoretical basis for HPA-targeted therapy of cervical cancer and metastatic LNs simultaneously.

\section{Materials and methods}

Patients. We retrospectively reviewed 102 consecutive patients with histologically confirmed cervical squamous cell cancer and well-documented clinical reports, who received standard surgery in the Second Affiliated Hospital of Zhengzhou
University, China, between January 2007 and December 2012. Among the patients, there were 53 cases with positive LNs (group A) and 49 negative cases (group B). In group A, the primary lesion and positive LNs were selected, while the primary and all LNs were selected in group B. Slices were secondly confirmed by experienced pathologists through routine pathological methods and no patients had undergone RT/chemotherapy or immunotherapy. The tumor stage was determined according to the 2011 FIGO clinical classification system for cervical cancer (21). Tumor differentiation was graded according to the World Health Organization (WHO) classification (22).

Of all cases, 29 were stage IA-IB and 73 were stage IIA, while 38 were well-differentiated and 64 were moderately to poorly differentiated. The complete follow-up data were obtained and the longest maturity was 60 months. Of all cases, 19 suffered a relapse, 12 succumbed to the disease, and the shortest survival period was 7 months. The survival period was calculated from the date of surgery, and the date of mortality or the last follow-up was recorded as the follow-up termination date. The follow-up deadline was December 30, 2012 and the median follow-up time was 56.5 months.

The study was conducted in accordance with the declaration of Helsinki, and with approval from the Ethics Committee of Zhengzhou University. Written informed consent was obtained from all participants.

Reagents and sample processing. Consecutive 4- $\mu$ m-thick tissue sections were cut from formalin-fixed and paraffin-embedded tissue samples for routine hematoxylin and eosin (HE) staining and HPA and cytokeratin (CK)19 immunohistochemical staining, respectively. CK19 is a squamous epithelial marker. Concentrated rabbit anti-human HPA1 antibody (1:150, sc-25825) was purchased from Santa Cruz Biotechnology, Inc. (Dallas, TX, USA). A ready-to-use mouse anti-human CK19 monoclonal antibody (MAB-0056), DAB kit, UltraSensitive ${ }^{\mathrm{TM}}$ SP IHC kit, citrate antigen repair fluid, and hematoxylin and eosin were purchased from Maixin.bio (Fuzhou, China).

Immunohistochemistry. Slices were cleaned, dried and wiped with Poly-L-Lysine solution, then set aside after heating at $55-60^{\circ} \mathrm{C}$ for $2 \mathrm{~h}$ in the oven. Sample sections measuring $4 \mu \mathrm{m}$ were boiled in citrate buffer liquid ( $\mathrm{pH} \mathrm{6.0)} \mathrm{for} \mathrm{antigen}$ repair. The endogenous peroxidases were blocked by rinsing the slides in 10 vol hydrogen peroxide $(3 \%)$. Slices were incubated with rabbit anti-human HPA monoclonal antibody (1:150, sc-25825) or mouse anti-human CK19 monoclonal antibody (1:150, MAB-0056) overnight at $4^{\circ} \mathrm{C}$ followed by a streptavidin-biotin-peroxidase complex compound at room temperature for $10 \mathrm{~min}$ and finally washed three times with phosphate-buffered saline (PBS) for 3 min each time. The peroxidase reaction was visualized with a diaminobenzidine (DAB) buffer. The slides were rinsed clean under running water and then counterstained in hematoxylin, dehydrated in 70,95 and $100 \%$ ethanol and xylene, and then mounted with a coverslip by neutral balsam. A negative control sample was obtained by replacing the primary antibody with PBS, while the positive control sample contained confirmed positive specimens. 
A
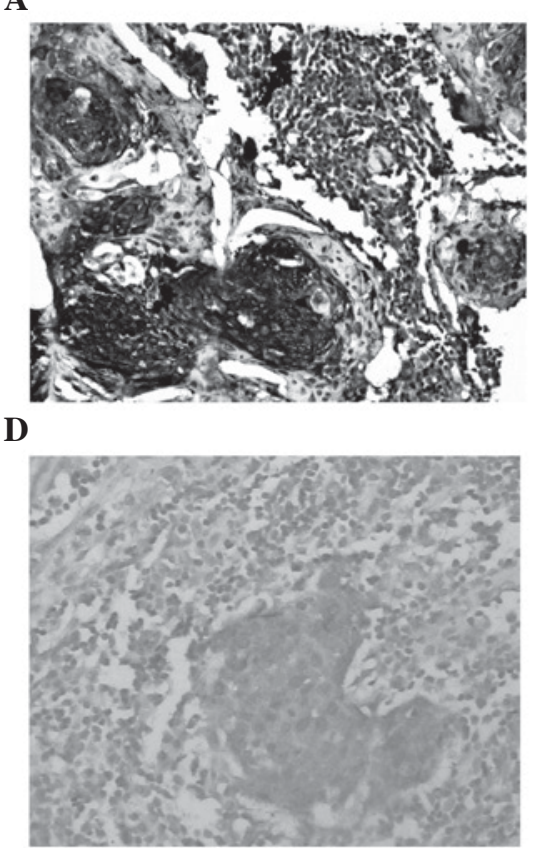

B

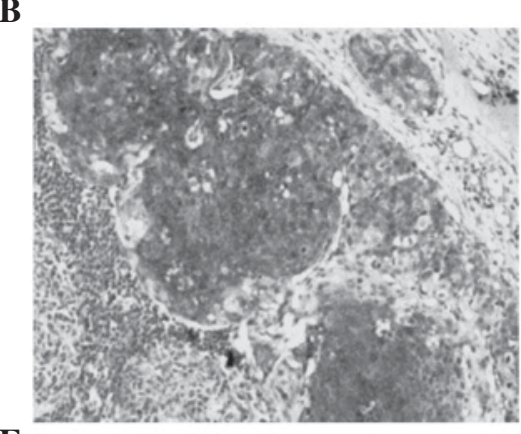

E

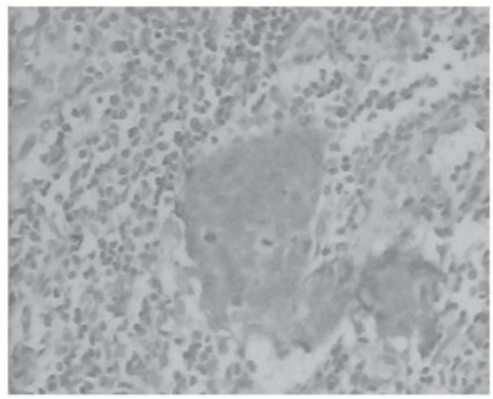

C
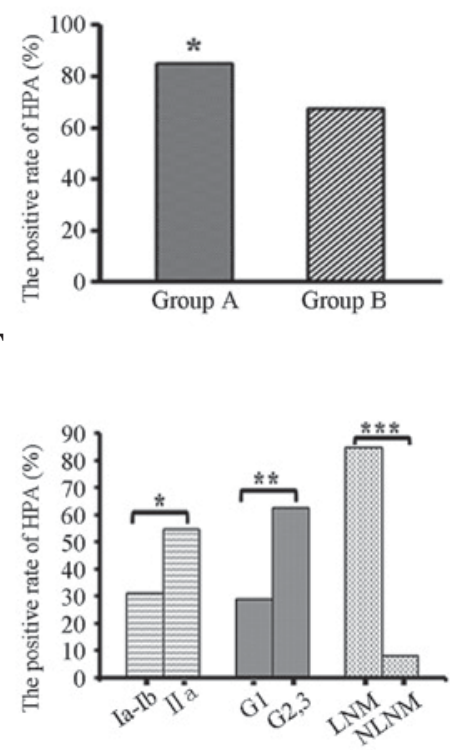

Figure 1. Expression of heparanase (HPA) in primary lesions and metastatic lymph nodes of cervical squamous cancer. (A) Intense positive immunohistochemical staining of HPA in primary lesion of cervical cancer; magnification, x200. (B) Positive immunohistochemical staining of HPA in metastatic lymph node; magnification, x400. (C) Expression rate of HPA in primary lesions of cervical cancer patients with positive lymph node metastasis (group A) was distinctly higher than that of non-metastatic cases. (D) Positive immunohistochemical staining of HPA in pathologically diagnosed non-metastatic lymph node; magnification, x400. (E) Positive immunohistochemical staining of cytokeratin 19 in consecutive section of sample in (D); magnification, $\mathrm{x}$ 400. (F) Correlation between positive rate of HPA in lymph nodes and clinicopathological features. LNM, lymph node metastasis; NLNM, no lymph node metastasis. "Comparison between two groups, $\mathrm{P}<0.05 ;{ }^{* *}$ comparison between two groups, $\mathrm{P}<0.005$; ${ }^{* * *}$ comparison between two groups, $\mathrm{P}<0.001$.

Determination of positive immunohistochemical results. Subsequently, the stained and coded sections were assessed by two pathologists blinded to the groups according to the combination of staining density and percentage of positive cells. Staining results were distributed through a 0 to 3 intensity scoring scale: 3 , brown/yellow granules in the cytoplasm; 2 , yellow granules; 1 or 0 , faint yellow granules or no granules, respectively. The positive ratio of cells (number of positive cells / number of total cells x 100\%) was calculated at high magnification: $6-25 \%, 1$ point; $26-50 \%, 2$ points; $51-75 \%$, 3 points; $\geq 75 \%, 4$ points. The final score was the product of the staining density score and the positive cell ratio score, and $\geq 3$ points was considered positive.

Statistical analysis. The results of immunohistochemical staining were expressed as the means \pm standard deviation. The expression of HPA was examined by the $\chi^{2}$ test. Survival rates were calculated using the Kaplan-Meier method and differences were examined using the log-rank test. Furthermore, the multivariate analysis was determined by the Cox proportional hazards model. These analyses were performed using SPSS version 11.0 (SPSS, Inc., Chicago, IL, USA). P<0.05 was considered to indicate a statistically significant difference.

\section{Results}

Expression of HPA in primary lesions and LNs of cervical squamous cancer. Positive HPA expression was detected in primary lesions and metastatic LNs as browny yellow or brown particles, which were located in the cytoplasm and membrane of cancer cells. The HPA level was significantly increased in primary lesions and metastatic LNs of cervical cancer (Fig. 1A and B). Pathologically diagnosed non-metastatic LNs also demonstrated positive staining in single cell or focal spots. For these samples, consecutive section CK19 immunohistochemical staining was conducted. Then pathologists determined whether there was LN involvement or not according to the HE and CK19 staining status. The location of positive CK19 staining was in accordance with HPA expression sites in HPA-positive LNs (Fig. 1D and E).

The expression rate of HPA in primary lesions of cervical cancer was $76.5 \%$ (78/102). Forty-five cases $(84.9 \%, 45 / 53)$ in group A demonstrated positive HPA expression in primary lesions and metastatic LNs, while in group B 33 cases $(67.3 \%$, $33 / 49$ ) were positive among the primary lesions and 4 cases $(8.2 \%, 4 / 49)$ among LNs. The positive expression of group A notably exceeded that in group $\mathrm{B}(\mathrm{P}<0.05$, Fig. 1C). Following immunohistochemical staining of HPA and CK19, the number of $\mathrm{LN}$ metastasis cases rose to 57.

Correlation between HPA expression and clinicopathological features of cervical squamous cancer. In terms of clinicopathological features, the expression rate of HPA in the LNs of stage IIA patients was distinctly higher that in stage IA-IB patients. In addition, the expression rate of LNs was higher in the moderate and low-differentiated tumors compared with that in well-differentiated tumors. Finally, patients with positive LN metastasis expressed higher levels of HPA than non-metastatic cases. All of these differences were statistically significant $(\mathrm{P}<0.05$, Fig. $1 \mathrm{~F})$. 
Table I. Cox regression analysis of prognostic factors in patients with cervical squamous carcinoma.

\begin{tabular}{|c|c|c|c|c|c|}
\hline Features & $\mathrm{B}$ & SE & RR & P-value & $95 \% \mathrm{CI}$ \\
\hline Clinical stage (IA-IB, IIA) & 1.132 & 0.378 & 1.323 & 0.067 & $0860-2.676$ \\
\hline Degree of differentiation & 1.027 & 0.339 & 1.218 & 0.073 & $0.854-2.904$ \\
\hline Lymph node involvement (negative, positive) & 1.942 & 0.451 & 1.636 & 0.039 & $1.203-3.351$ \\
\hline Expression of HPA (negative, positive) & 1.561 & 0.362 & 1.473 & 0.047 & $1.188-2.975$ \\
\hline
\end{tabular}

B, regression coefficient; SE, standard error; RR, relative risk; CI, confidence interval; HPA, heparanase.
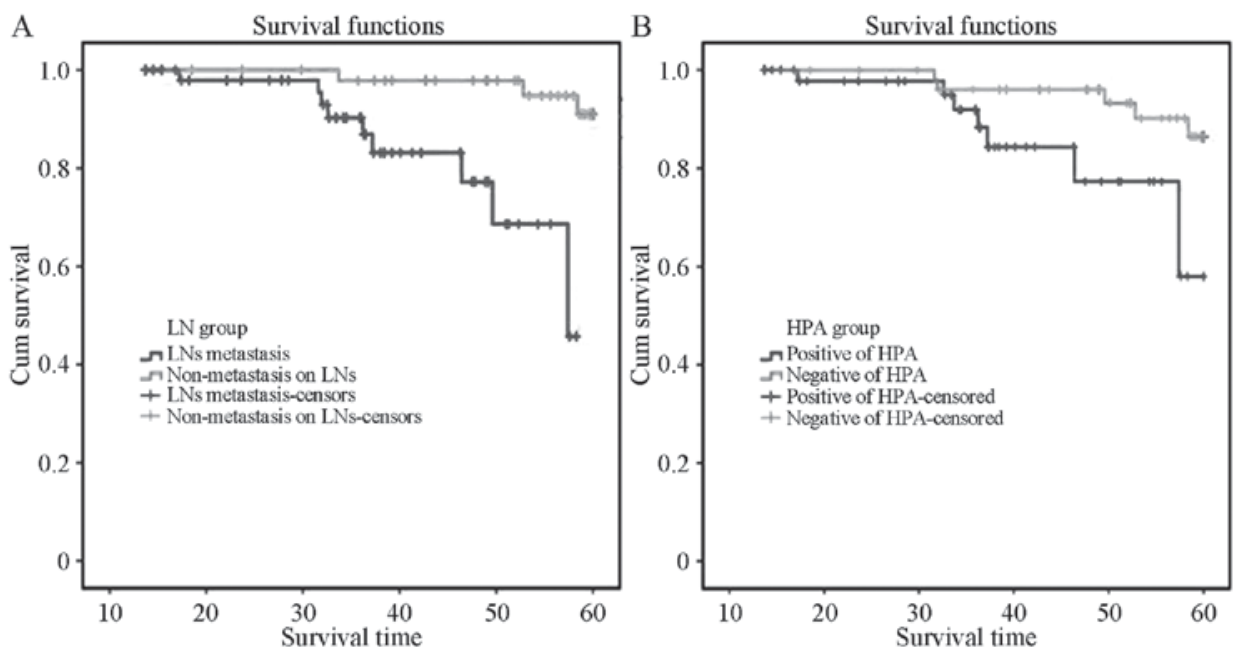

Figure 2. Analysis of the expression of heparanase (HPA) in lymph nodes (LNs) and the survival of cervical squamous cancer patients. (A) Prognosis of patients with LN involvement was poor. (B) Positive expression of HPA is involved in the poor prognosis of cervical cancer patients.

Correlation between expression of HPA in LNs and survival of cervical squamous cancer patients. The 5-year overall survival rate was $73.3 \%$ and the median overall survival (MOS) was 49.0 months. The MOS of the 53 patients with positive LN metastasis in group A was 36.0 months, while in group B, the MOS was 58.5 months. Kaplan-Meier survival analysis indicated that the MOS of the positive metastasis group was distinctly lower than that of the non-metastatic patients, and the difference was statistically significant $(\mathrm{P}=0.023)$ by log-rank test (Fig. 2A). The MOS of the 49 patients with positive HPA expression was 38.5 months, while the MOS of the 53 negative patients was 57.0 months, indicating that the MOS of cervical squamous cancer patients with positive HPA expression was distinctly lower than that of negative patients ( $\mathrm{P}=0.04$, Fig. $2 \mathrm{~B}$ ). Single-factor Cox regression analysis suggested that clinical staging, differentiation degree, LN metastasis and expression of HPA notably affected patient prognosis $(\mathrm{P}<0.05)$; moreover, multiple-factor Cox model analysis indicated that LN metastasis and the expression of HPA were independent risk factors affecting the prognosis of cervical cancer patients $(\mathrm{P}<0.05$, Table I).

\section{Discussion}

Our study demonstrated that the level of HPA expression in pathologically diagnosed metastatic retroperitoneal LNs was as high as that in primary lesions. The expression rate of HPA in LNs of cervical squamous cancer patients was much higher in patients of stage IIA than in those of stage IA-IB. In addition, moderate or poorly differentiated cases expressed more HPA than well-differentiated cases. The long-term survival rate of patients with positive HPA expression was notably lower, which indicated that the expression of HPA was an independent risk factor affecting the prognosis of cervical cancer patients. Hence, our results further confirmed that HPA was abnormally highly expressed in cervical cancer patients and that metastatic retroperitoneal LNs were homologous with primary lesions of cervical squamous cancer, indicating that the abnormal expression of HPA played a significant role in LN metastasis of cervical cancer and was involved in the course of retroperitoneal LN metastasis, thereby facilitating distant metastasis in cervical cancer patients and affecting their long-term survival.

HPA plays its biological role via its glycosidase activity, which is involved in degrading heparan sulphate (HS) in the extracellular matrix (ECM), and in an enzymatic activityindependent manner (23). HS is a primary component at the interface between virtually every eukaryotic cell and its ECM, and is vital in maintaining biological processes in sick and healthy individuals. HS combines with proteoglycan to form heparan sulfate proteoglycans as three-dimensional structures of the matrix to maintain the connection of normal cells. In certain cases, they not only provide a storage depot for heparin-binding molecules in the cell microenvironment, but also decisively regulate their accessibility, function 
and mode of action by connecting with receptors as signal molecules $(24,25)$. Overexpression of HPA in cervical cancer tissues degrades the side chain of heparan sulfate glycosaminoglycan (HS-GAG) connected on perlecan located on the surface of the ECM $(26,27)$, which results in the release of multiple cytokines and growth factors that bond to HS-GAG, thus facilitating the transfer of cervical cancer cells to the lymph vessels.

HPA plays a significant role in promoting the formation of lymph vessels, which has been reported to be one of the main transfer pathways of malignant cells. Tumor cells produce and release growth factors that are associated with the formation of lymph vessels, including vascular endothelial growth factor (VEGF)-C and VEGF-D, hence inducing formation and transfer (28). High levels of VEGF-C may be detected in the serum of cervical cancer patients, and the expression of VEGF-C in samples of patients with positive LN metastasis is extraordinarily high in comparison with that of non-metastatic patients $(29,30)$. By inhibiting the function of the upstream regulatory factors of VEGF-C, the expression of VEGF-C and its effect of inducing angiogenesis is suppressed (31). VEGF-C and VEGF, together with the essential lymphangiogenesis factor Proxl, may be involved in the formation of early lymph vessels during the progression of cervical neoplasia, which explains the reason why LN involvement may even be detected in certain early-stage cervical cancers. Although experimental studies that identify the function of HPA in promoting the secretion of VEGF are poorly reported, melanoma, breast and prostate cancer cells with overexpression of HPA cause the level of VEGF-C to increase by 3-5 times, and promote angiogenesis of transplant tumors. Conversely, the silencing of HPA genes reduces the VEGF-C level (32). The present study revealed that the expression level of HPA in primary lesions of cervical cancer patients with LN metastasis was notably higher than that in non-metastatic cases, which supports the hypothesis that HPA promotes LN metastasis from the point of view of clinicopathology.

When evaluating the role HPA plays in tumor sentinel LN dissemination, Dafni et al (33) observed that Eb tumors with high expression of HPA increased extravasation, interstitial convection and lymphatic drain of the contrast material using dynamic contrast-enhanced magnetic resonance imaging (MRI), and changes in MR contrast enhancement were detectable when only a few Eb cells were identified near and within the nodes. These authors demonstrated that HPA of the tumor cells could promote the release of vascular endothelial growth factor, which triggered secondary angiogenesis during tumor cell cloning in LNs. Our study identified that metastatic LNs have the same HPA expression level as the original tumor site, which theoretically supported their theory. Our results revealed that the prognosis of patients with positive HPA in LNs was poorer than that of patients without HPA expression; this may be due to the promotion of angiogenesis by HPA, which facilitated the formation of tumor cloning and shortened the formation time of the secondary tumor, and ultimately constitutes a threat to long-term survival in patients (33).

Notably, we observed that out of the 49 clinicopathologically diagnosed patients without metastatic LNs, 4 cases were positive for immunostaining of HPA and CK19, and these cases were identified by an experienced pathologist to have micro-metastasis of the LNs. CK19 is the most common diagnostic marker of LN micro-metastasis in gynecological malignant tumors $(7,34,35)$. Our study revealed that, in the primary lesion and metastatic LNs, the positive expression rate of HPA was over $80 \%$ and no less than the rate of CK19, which supported the theory that HPA could be a promising biomarker of LN metastasis in cervical cancer.

For the first time, we confirm that overexpression of HPA may be detected in metastatic retroperitoneal LNs of cervical cancer, and may be involved in lymphatic transfer of cervical cancer and further affect patient prognosis. Our findings support the theory that HPA could be a biomarker for the diagnosis of LN micro-metastasis in cervical cancer, offer a significant basis for the development of positron emission tomography tracers, and provide a promising target marker for the treatment of patients with LN metastasis.

\section{Acknowledgements}

The present study was supported by the National Natural Science Foundation of China (Beijing, China; grant no. 81341065), the Henan International Cooperation Project (Zhengzhou, China; grant no. 134300510047) and the Natural Science Research Program of the Education Department of Henan Province (Zhengzhou, China; grant no. 12A320024).

\section{References}

1. Ho CM, Chien TY, Huang SH, Wu CJ, Shih BY and Chang SC: Multivariate analysis of the prognostic factors and outcomes in early cervical cancer patients undergoing radical hysterectomy. Gynecol Oncol 93: 458-464, 2004.

2. Slama J, Dundr P, Dusek L and Cibula D: High false negative rate of frozen section examination of sentinel lymph nodes in patients with cervical cancer. Gynecol Oncol 129: 384-388, 2013.

3. Quinn MA, Benedet JL, Odicino F, Maisonneuve P, Beller U, Creasman WT, Heintz AP, Ngan HY and Pecorelli S: Carcinoma of the cervix uteri. FIGO 26th annual report on the results of treatment in gynecological cancer. Int J Gynaecol Obstet 95 (Suppl 1): S43-S103, 2006.

4. Ditto A,Martinelli F,Lo Vullo S, Reato C, Solima E, Carcangiu M, Haeusler E, Mariani L, Lorusso D and Raspagliesi F: The role of lymphadenectomy in cervical cancer patients: the significance of the number and the status of lymph nodes removed in 526 cases treated in a single institution. Ann Surg Oncol 20: 3948-3954, 2013.

5. Slama J, Fischerova D, Pinkavova I, Zikan M and Cibula D: Human papillomavirus DNA presence in pelvic lymph nodes in cervical cancer. Int J Gynecol Cancer 20: 126-132, 2010.

6. Park JS, Namkoong SE, Han SK, Nha DJ, Lee HY and Kim SJ: Comparison of L1 consensus primers with E6 type specific primers for detection of human papillomaviruses in paraffin sections of cervical neoplasia. J Korean Med Sci 8: 60-67, 1993.

7. Noventa M, Ancona E, Cosmi E, Saccardi C, Litta P, D'Antona D, Nardelli GB and Gizzo S: Usefulness, methods and rationale of lymph nodes HPV-DNA investigation in estimating risk of early stage cervical cancer recurrence: a systematic literature review. Clin Exp Metastasis 31: 853-867, 2014.

8. Forman D, de Martel C, Lacey CJ, Soerjomataram I, Lortet-Tieulent J, Bruni L, Vignat J, Ferlay J, Bray F, Plummer M and Franceschi S: Global burden of human papillomavirus and related diseases. Vaccine 30 (Suppl 5): F12-F23, 2012.

9. Tjalma WA, Van Waes TR, Van den Eeden LE and Bogers JJ: Role of human papillomavirus in the carcinogenesis of squamous cell carcinoma and adenocarcinoma of the cervix. Best Pract Res Clin Obstet Gynaecol 19: 469-483, 2005.

10. Garland SM: Can cervical cancer be eradicated by prophylactic HPV vaccination? Challenges to vaccine implementation. Indian J Med Res 130: 311-321, 2009. 
11. Woodman CB, Collins SI and Young LS: The natural history of cervical HPV infection: unresolved issues. Nat Rev Cancer 7: 11-22, 2007.

12. Hoste G, Vossaert K and Poppe WA: The clinical role of HPV testing in primary and secondary cervical cancer screening. Obstet Gynecol Int 2013: 610373, 2013.

13. Hirshoren N, Bulvik R, Neuman T, Rubinstein AM, Meirovitz A and Elkin M: Induction of heparanase by HPV E6 oncogene in head and neck squamous cell carcinoma. J Cell Mol Med 18: 181-186, 2014.

14. Baraz L, Haupt Y, Elkin M, Peretz T and Vlodavsky I: Tumor suppressor $\mathrm{p} 53$ regulates heparanase gene expression. Oncogene 25: 3939-3947, 2006.

15. Adams DH and Shaw S: Leucocyte-endothelial interactions and regulation of leucocyte migration. Lancet 343: 831-836, 1994.

16. Blotnick S, Peoples GE, Freeman MR, Eberlein TJ and Klagsbrun M: T lymphocytes synthesize and export heparin-binding epidermal growth factor-like growth factor and basic fibroblast growth factor, mitogens for vascular cells and fibroblasts: differential production and release by CD4+ and CD8+ T cells. Proc Natl Acad Sci USA 91: 2890-2894, 1994.

17. Vlodavsky I, Eldor A, Haimovitz-Friedman A, Matzner Y, Ishai-Michaeli R, Lider O, Naparstek Y, Cohen IR and Fuks Z: Expression of heparanase by platelets and circulating cells of the immune system: possible involvement in diapedesis and extravasation. Invasion Metastasis 12: 112-127, 1992.

18. Pisano C, Vlodavsky I, Ilan N and Zunino F: The potential of heparanase as a therapeutic target in cancer. Biochem Pharmacol 89: 12-19, 2014.

19. Shinyo Y, Kodama J, Hongo A, Yoshinouchi M and Hiramatsu Y: Heparanase expression is an independent prognostic factor in patients with invasive cervical cancer. Ann Oncol 14: 1505-1510, 2003

20. Zeng C, Ke ZF, Luo WR, Yao YH, Hu XR, Jie W, Yin JB and Sun SJ: Heparanase overexpression participates in tumor growth of cervical cancer in vitro and in vivo. Med Oncol 30: 403, 2013

21. Prat J; FIGO Committee on Gynecologic Oncology: Staging classification for cancer of the ovary, fallopian tube, and peritoneum. Int J Gynaecol Obstet 124: 1-5, 2014.

22. Kurman RJ, Carcangiu ML, Herrington CS and Young RH (eds): WHO Classification of Tumours of the Female Reproductive Organs. In: WHO/IARC Classification of Tumours.Vol 6. 4th edition. IARC, Lyon, 2014

23. Vlodavsky I, Elkin M and Ilan N: Impact of heparanase and the tumor microenvironment on cancer metastasis and angiogenesis: basic aspects and clinical applications. Rambam Maimonides Med J 2: e0019, 2011.

24. Kim SH, Turnbull J and Guimond S: Extracellular matrix and cell signalling: the dynamic cooperation of integrin, proteoglycan and growth factor receptor. J Endocrinol 209: 139-151, 2011.
25. Barbouri D, Afratis N, Gialeli C, Vynios DH, Theocharis AD and Karamanos NK: Syndecans as modulators and potential pharmacological targets in cancer progression. Front Oncol 4: 4 , 2014.

26. Hasengaowa, Kodama J, Kusumoto T, Shinyo Y, Seki N, Nakamura K, Hongo A and Hiramatsu Y: Loss of basement membrane heparan sulfate expression is associated with tumor progression in endometrial cancer. Eur J Gynaecol Oncol 26: 403-406, 2005.

27. Kodama J, Shinyo Y, Hasengaowa, Kusumoto T, Seki N, Nakamura K, Hongo A and Hiramatsu Y: Loss of basement membrane heparan sulfate expression is associated with pelvic lymph node metastasis in invasive cervical cancer. Oncol Rep 14: 89-92, 2005.

28. Ozasa R, Ohno J, Iwahashi T and Taniguchi K: Tumor-induced lymphangiogenesis in cervical lymph nodes in oral melanoma-bearing mice. J Exp Clin Cancer Res 31: 83, 2012.

29. Biedka M, Makarewicz R, Marszałek A, Sir J, Kardymowicz H and Goralewska A: Labeling of microvessel density, lymphatic vessel density and potential role of proangiogenic and lymphangiogenic factors as a predictive/prognostic factors after radiotherapy in patients with cervical cancer. Eur J Gynaecol Oncol 33: 399-405, 2012.

30. Liu H, Xiao J, Yang Y, Liu Y, Ma R, Li Y, Deng F and Zhang Y: COX-2 expression is correlated with VEGF-C, lymphangiogenesis and lymph node metastasis in human cervical cancer. Microvasc Res 82: 131-140, 2011.

31. Liu D, Li L, Zhang XX, Wan DY, Xi BX, Hu Z, Ding WC, Zhu D, Wang XL, Wang W, et al: SIX1 promotes tumor lymphangiogenesis by coordinating TGF $\beta$ signals that increase expression of VEGF-C. Cancer Res 74: 5597-5607, 2014.

32. Cohen-Kaplan V, Naroditsky I, Zetser A, Ilan N, Vlodavsky I and Doweck I: Heparanase induces VEGF C and facilitates tumor lymphangiogenesis. Int J Cancer 123: 2566-2573, 2008.

33. Dafni H, Cohen B, Ziv K, Israely T, Goldshmidt O, Nevo N, Harmelin A, Vlodavsky I and Neeman M: The role of heparanase in lymph node metastatic dissemination: dynamic contrast-enhanced MRI of Eb lymphoma in mice. Neoplasia 7: 224-233, 2005.

34. Wang HY, Sun JM, Lu HF, Shi DR, Ou ZL, Ren YL and Fu SQ: Micrometastases detected by cytokeratin 19 expression in sentinel lymph nodes of patients with early-stage cervical cancer. Int J Gynecol Cancer 16: 643-648, 2006.

35. Nagai T, Niikura H, Okamoto S, Nakabayashi K, Matoda M, Utsunomiya $\mathrm{H}$, Nagase $\mathrm{S}$, Watanabe $\mathrm{M}$, Takeshima $\mathrm{N}$ and Yaegashi N: A new diagnostic method for rapid detection of lymph node metastases using a one-step nucleic acid amplification (OSNA) assay in endometrial cancer. Ann Surg Oncol 22: 980-986, 2015. 